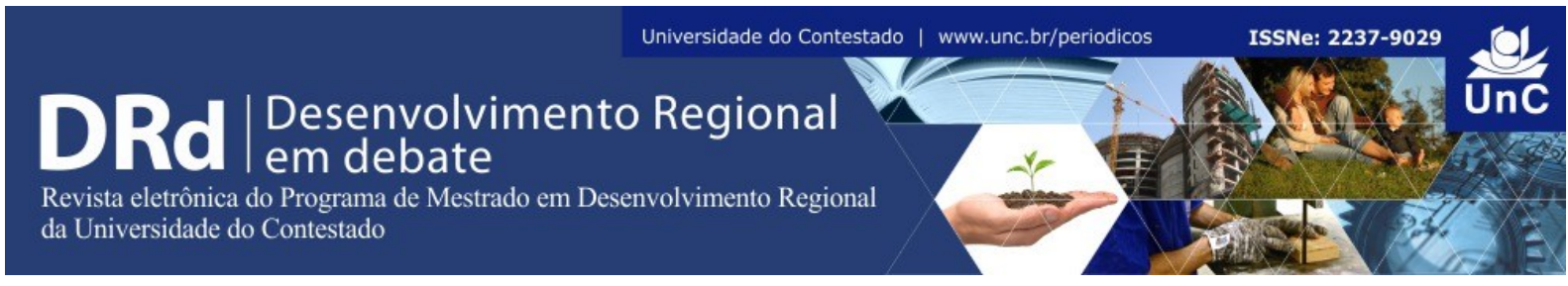

\title{
ECONOMÍA CREATIVA, DINÁMICAS LOCALES Y GOBERNAZA EN ENTORNOS METROPOLITANOS: EL CASO DE LA REGIÓN METROPOLITANA DE SALVADOR DE BAHIA ${ }^{1}$
}

Mariña Pose García

\begin{abstract}
RESUMEN
La economía creativa se está configurando como un sector estratégico en el desarrollo socioeconómico de los países occidentales. Alineado a esta perspectiva, Brasil ha reconocido la importancia de la economía creativa como uno de los ejes transformadores del proceso de cambio que está teniendo lugar en el país (SIERRA, 2015). Con base en una revisión teórica sobre gobernanza y economía creativa, el artículo analiza los sectores creativos en la Región Metropolitana de Salvador de Bahía (Brasil), considerando tanto su peso en la economía urbana como la lógica espacial que determina su distribución territorial. Los objetivos centrales del presente artículo, que en sus dos primeros apartados pretende revisar de forma crítica la relación entre los conceptos de gobernanza urbana, economía creativa y desarrollo territorial, son aportar algunas claves sobre la distribución territorial de las industrias culturales. Nos centraremos sobre todo en la habitual referencia a la concentración espacial de estas actividades, y mostraremos un mapa de la economía creativa en la Región Metropolitana de Salvador de Bahía (Brasil). El documento tiene como objetivo contribuir a una comprensión más amplia sobre esta relación en regiones metropolitanas.
\end{abstract}

Palabras clave: Economía creativa. Gobernanza. Desarrollo urbano.

\section{CREATIVE ECONOMY, LOCAL DYNAMICS AND GOVERNANCE IN METROPOLITAN AREAS: THE CASE OF THE METROPOLITAN REGION OF SALVADOR DE BAHIA}

\begin{abstract}
The creative economy is emerging as a strategic sector in the socio-economic development of Western countries. Aligned to this perspective, Brazil has recognized the importance of the creative economy as one of the axes transformers of the change process that is taking place in the country (SIERRA, 2015). Based on a theoretical review of governance and creative economy, the article analyzes the creative industries in the metropolitan region of Salvador de Bahia (Brazil), considering both their weight in the urban economy and spatial logic that determines its territorial distribution. The main objectives of this Article, which in its first two paragraphs aims to review critically the relationship between the concepts of urban governance, creative economy and regional development, are providing clues about the

\footnotetext{
${ }^{1}$ Este artículo, en su primera versión, fue presentado en el II Congreso Internacional SETED-ANTE, en Santiago de Compostela, España (julio, 1-3 - 2015).

${ }^{2}$ Doutora em Geografia pela Universidade de Santiago de Compostela. Atua no Programa de Pós-Graduação e Geografia. Instituto de Geociências. Universidade Federal da Bahia. Brasil. E-mail: marinhapose@gmail.com
}

DRd - Desenvolvimento Regional em debate (ISSNe 2237-9029) 
territorial distribution of cultural industries. We will focus especially on the usual reference to the spatial concentration of these activities, and show a map of the creative economy in the metropolitan region of Salvador de Bahia (Brazil). The document aims to contribute to a wider understanding of this relationship in metropolitan regions.

Keywords: Creative economy. Governance. Urban development.

\section{INTRODUCCIÓN}

La relación entre creatividad y desarrollo urbano, nuevas jerarquías urbanas y el reconocimiento de la importancia creciente de las actividades culturales y creativas en la economía y en el desarrollo territorial, son solamente algunas de las principales variantes del interés y de los múltiples enfoques que suscita este tema. El presente artículo se enmarca dentro de este campo de estudio a partir del análisis de dos factores relacionados: gobernanza urbana y economía creativa. El documento tiene como objetivo contribuir a una comprensión más amplia sobre esta relación en regiones metropolitanas.

Desde hace ya más de una década se viene atribuyendo un valor estratégico a la economía creativa al entender que puede contribuir a estimular la creación de empleo y a promover nuevos modelos de desarrollo territorial. De hecho, la importancia que adquiere la economía creativa en el mundo ha ido aumentando en los últimos años, con una tasa media de crecimiento anual durante el período 2002-2011 del 8,8\% (UNCTAD, 2013).

Con este contexto general de referencia se analizan los sectores creativos en la Región Metropolitana de Salvador de Bahía (en adelante, RMS), considerando tanto su peso en la economía urbana como la lógica espacial que determina su distribución territorial. Los objetivos centrales del presente artículo, que en sus dos primeros apartados pretende revisar de forma crítica la relación entre los conceptos de gobernanza urbana, economía creativa y desarrollo territorial, son aportar algunas claves sobre la distribución territorial de las industrias culturales. Nos centraremos sobre todo en la habitual referencia a la concentración espacial de estas actividades, y mostraremos un mapa de la economía creativa en la RMS. Para ello, se seguirá el modelo realizado previamente por Méndez et al. (2012) en la Región Metropolitana de Madrid, con el fin de posibilitar la realización de comparaciones con estudios similares realizados en otros países. Para llevar a cabo la investigación se elaboró una amplia base de datos estadísticos mediante la utilización de diversas fuentes oficiales, junto con diverso material documental sobre las iniciativas desarrolladas en la región: estudios publicados en los últimos años y noticias recogidas en la prensa local y/o regional

Es importante destacar las limitaciones de las que adolece este trabajo relacionadas con las fuentes estadísticas y su nivel de desagregación. El Instituto Brasileiro de Geografia e Estatística (IBGE) recoge el número de trabajadores ocupados en las diferentes industrias culturales según el CNAE2.0, pero muchas veces estos sectores incluyen puestos de trabajo que no son específicamente creativos y que conllevan tareas rutinarias de apoyo a otros sectores, como puestos de trabajo relacionados con la logística o la reparación de equipamiento tecnológico. Las dificultades de desagregación de los datos pueden conllevar una sobrevaloración de su importancia. También existen limitaciones en el análisis

DRd - Desenvolvimento Regional em debate (ISSNe 2237-9029) 
estadístico, pues hemos tenido que restringirnos a la subcategoría de actividades que proporciona el IGBE en vez de seguir el modelo establecido por los diversos informes de la UNCTAD (2008, 2010 y 2013), lo que facilitaría la realización de comparaciones con trabajos similares en otras áreas. Las dificultades de comparación de los datos, sus definiciones inconsistentes y las diferentes clasificaciones de las industrias creativas dificulta la evaluación de la contribución real de las industrias creativas y culturales en las ciudades, regiones y economías nacionales.

\section{ECONOMÍA CREATIVA Y DESARROLLO URBANO}

Economía creativa, economía cultural, industrias creativas, industrias culturales, ciudades creativas... son conceptos utilizados para definir la relación entre la cultura y la economía, y reflejan la falta de acuerdo sobre su definición. La economía creativa sería una fusión entre la economía de la cultura y la economía del conocimiento, y tiene en su centro las industrias creativas, estos es, sectores con gran carga de capital intelectual, lo que centra la discusión en un abanico de sectores abarcando cultura y ciencia y tecnología (FONSECA, 2014).

El origen del concepto economía creativa se sitúa en el término de industrias creativas, a su vez inspirado en el proyecto gubernamental Creative Nation (AUSTRALIA, 1994), que proponía la búsqueda de la identidad cultural australiana. Entre otros elementos, el proyecto promulgaba la importancia del trabajo creativo, su contribución para la economía del país y el papel de las nuevas tecnologías de la información y la comunicación como aliadas de la política cultural.

En el ámbito académico, uno de los principales pensadores en este campo ha sido el británico John Howkins, autor del libro The Creative Economy: How People Make Money From Ideas (2001). Howkins (2001) fue uno de los primeros en señalar la importancia de la creatividad en el desarrollo económico moderno y, aunque reconoce que las relaciones entre la creatividad y la economía no suponen una novedad, la naturaleza y la extensión de las relaciones para crear valor y riqueza sí son características principales del nuevo paradigma económico. Dicha riqueza estaría relacionada con el sector de la economía que produce bienes en forma de propiedad intelectual (patentes, copyrights, trademarks y designs).

Desde el 2008, la Conferencia de las Naciones Unidas sobre Comercio y Desarrollo (UNCTAD) y el Programa para el Desarrollo de las Naciones Unidas (PNUD), han elaborado tres informes sobre la economía creativa (Informes 2008, 2010 y 2013). Los informes de 2008 y 2010 definían a la "economía creativa" como las actividades relacionadas con la creatividad cultural y la innovación en el núcleo de lo que comúnmente se denomina "industrias culturales", "industrias creativas" o, de hecho, "industrias culturales y creativas". Las ediciones de 2008 y 2010, tal y como se reconoce en el informe 2013, proporcionaron un marco sólido para entender la economía creativa como un sector económico transversal, sobre todo en lo referente al comercio internacional de bienes y servicios culturales y creativos. En el Informe 2010 se constataba que, "adecuadamente nutrida, la creatividad impulsa la cultura, infunde un desarrollo centrado en el hombre y constituye el ingrediente clave para la creación de empleo, la innovación y el comercio, al tiempo que contribuye a la inclusión social, la

DRd - Desenvolvimento Regional em debate (ISSNe 2237-9029) 
diversidad cultural y la sostenibilidad ambiental". Así, el Informe coloca en una nueva posición el capital social en la cúspide de un nuevo enfoque del desarrollo territorial. El cambio transformador, por tanto, se entiende o se encuentra en la persona, en lo local... Así lo reafirma el Informe 2013 al constatar que "[...] liberar el potencial de la economía creativa implica promover la creatividad general de las sociedades, afirmando la identidad distintiva de los lugares donde prospera y se agrupa, mejorando la calidad de vida, la imagen y el prestigio local, y fortaleciendo los recursos para imaginar nuevos futuros diversos" (UNCTAD, 2013, p. 15).

El informe del 2013, además de avanzar en el reconocimiento de la innovación y la creatividad humana, tanto a nivel individual como grupal, como los motores fundamentales de estas industrias y en situarlas como la auténtica riqueza de las naciones del siglo XXI, plantea, por primera vez, la importancia de las economías locales para fomentar la economía creativa y aporta la idea de que no hay una única economía creativa, sino una multitud de economías creativas independientes e interconectadas entre sí: "[...] pese a la importancia de la intervención de políticas a escala nacional, está claro que la siguiente frontera de la generación de conocimientos se basa en comprender las interacciones, particularidades y políticas en el plano local y en cómo promover la economía creativa de forma práctica en comunidades, ciudades y regiones del mundo en desarrollo" (UNCTAD, 2013, p. 10).

En Brasil, desde que el gobierno de Lula da Silva (2003-2010) iniciara una política integral de Estado en materia de cultura y desarrollo, y sentara las bases de un Sistema Nacional de Cultura (SNC) integrado, el país ha ido avanzando en el reconocimiento de la importancia estratégica de la cultura en el desarrollo socio-económico del país, un campo tradicionalmente abandonado en la mayoría de los países de la región (SIERRA, 2015).

El Ministerio de Cultura BR brasileño define la economía creativa como la suma de todos los sectores cuyas "[...] cujas atividades produtivas têm como processo principal um ato criativo gerador de um produto, bem ou serviço, cuja dimensão simbólica é determinante do seu valor, resultando em produção de riqueza cultural, econômica e social" (BRASIL, 2011. p. 22). Con esta definición, el gobierno brasileño reconoce la importancia de la cultura como factor estratégico para la generación de riqueza.

Un análisis de la literatura actual sobre economía creativa, permite identificar al menos tres características comunes sobre este tipo de actividades consideradas relevantes para el desarrollo urbano.

En primer lugar, se trata de actividades intensivas en conocimiento con creciente demanda internacional y capacidad de generar valor añadido, lo que parece asegurar su capacidad para generar empleo en el próximo futuro (MICHELINI; MÉNDEZ, 2012). Una parte al menos de las actividades creativas - las relacionadas con el patrimonio y las industrias culturales- generan beneficios que no pueden medirse únicamente en base a los precios del mercado, sino que se relaciona con la afirmación de la identidad territorial y una imagen de marca que puede generar efectos multiplicadores sobre otras, tal como ocurre con el turismo cultural (CARAVACA et al., 2013; GARRIDO, 2002).

En segundo lugar, son actividades que requieren de recursos humanos con elevado nivel formativo, que suelen asociarse con empleos de calidad y la formación de lo que Florida (2002) denominó la clase creativa, esto es, una nueva clase socioeconómica formada por

DRd - Desenvolvimento Regional em debate (ISSNe 2237-9029) 
científicos, ingenieros, profesores de universidad, músicos, diseñadores, arquitectos, e incluso bohemios, cuya función económica es crear nuevas ideas, nuevas tecnologías o nuevos contenidos culturales. La hipótesis subyacente de la clase creativa es que la presencia y concentración de bohemios en un área crea un ambiente de entorno que atrae a otros tipos de talentos o grandes capitales humanos. La presencia de ese capital humano, a su vez atrae y genera industrias innovadoras de base tecnológica. Tal y como afirma el autor, las personas creativas tienden a concentrarse en territorios con determinadas características, como la tecnología, el talento y la tolerancia. En la misma línea, Clifton y Cooke (2009) sostienen que los lugares con mayor balance de personas con estudios superiores son, en general, más innovadores y crecen más rápidamente y con firmeza en el tiempo.

En tercer lugar, Michelini y Méndez (2012) señalan que los recursos que utilizan las economías creativas son, a menudo, intangibles, lo que limita el riesgo de deslocalización empresarial en busca de áreas con menores costos respecto de otro tipo de actividades más móviles. Relacionado con esta tesis, Caravaca et al. (2013) afirma que el interés despertado en los gobiernos locales por este tipo de actividades es lógico, puesto que el crecimiento de las actividades que la conforman está en buena parte sustentado en la existencia de recursos patrimoniales, entre los que cabe destacar el conocimiento y la creatividad, recursos muy específicos, lo que los hace especialmente interesantes para promover procesos de desarrollo territorial (RALLET, 1995, sacado de CARAVACA et al., 2013). En este sentido, las diferentes capacidades de los gobiernos locales para poner en valor sus recursos intangibles y generar valor añadido, puede convertirse en un factor de nuevas desigualdades interurbanas (MICHELINI; MÉNDEZ, 2012).

Existen tres factores presentes de manera más o menos variable en la literatura internacional sobre las economías creativas: su tendencia a la concentración, visible a diferentes escalas, su propensión a la formación de clusters y la existencia de trayectorias locales, ligadas a las herencias y recursos específicos de cada ciudad (FOORD, 2008; CLIFTON; COOKE, 2009; LAZARETTI et al. 2008; MICHELIN; MÉNDEZ, 2012).

Ford (2008) añade que el crecimiento de la industria creativa se está produciendo a nivel local y no tanto a nivel nacional, lo que indica no sólo la alta concentración de empresas creativas en las ciudades, sino también la importancia competitiva de las ciudades particularmente creativas (ciudades con más alta que los niveles nacionales de empleo creativo) Por ejemplo, el nivel indicado de empleo creativo de la industria en Austria fue del 4 por ciento, pero el 14 por ciento en Viena; 3 por ciento en Alemania, pero el 8 por ciento en Berlín.

No obstante, el estudio realizado por Méndez et al. (2012) en diversos municipios españoles sostiene que, en algunas ciudades, la economía creativa da lugar a diferentes lógicas de organización espacial, relativizando la presencia de esos factores. Las razones hay que buscarlas en la superposición de múltiples variables de localización junto con la singularidad de cada espacio urbano, debido a trayectorias locales específicas. En el marco de este debate, Lazaretti et al. (2009), a partir de un estudio comparado entre España e Italia, sostiene que los patrones de distribución de las industrias creativas y clusters creativos varían entre países. Además, el nivel de influencia de esos patrones es variable y ninguno por sí solo es decisivo. Para la autora, dichos patrones están vinculados a la cultura, la creatividad, la innovación y el desarrollo local. En particular, la presencia de herencia cultural y artística en 
el territorio es una cuestión importante y una de las bases para el desarrollo de los distritos culturales y clusters.

Otra supuesto sobre el comportamiento de las economías creativas en debate, es el hecho de que se trata de actividades relativamente inmunes a los ciclos económicos $\mathrm{y}$, por consiguiente, al impacto de la actual crisis económica. Sin embargo, estudios recientes en Reino Unido (REID et al, 2010), evidencian que la disminución del empleo y la contratación en los sectores de la economía creativa debido a una bajada del consumo de este tipo de bienes y servicios. Foord (2008) sostiene esta idea al considerar que el crecimiento del empleo en la economía creativa ha comenzado a frenar, lo que sugiere que las industrias creativas están subsumidas en una industria de conocimiento más importante y amplia.

No obstante, el valor estratégico que ahora se les atribuye en numerosos estudios y documentos oficiales considera sobre todo criterios de índole cualitativa que van más allá de esas simples cifras (MÉNDEZ et al., 2012). La UNCTAD (2010) afirma "adecuadamente nutrida, la creatividad impulsa la cultura, infunde un desarrollo centrado en el hombre y constituye el ingrediente clave para la creación de empleo, la innovación y el comercio, al tiempo que contribuye a la inclusión social, la diversidad cultural y la sostenibilidad ambiental" (UNCTAD, 2010, p. 483)

A estas características de las economías creativas se le añaden otras como su potencial para generar innovación y la revalorización de las áreas donde se asientan. Además, se afirma que atraen salarios altos, tienden a ser respetuosas con el medio ambiente y suscitan cuestiones políticas y sociales específicas que las otras industrias no plantean (UNCTAD, 2013).

Resultado de esa indefinición de la economía creativa, es la falta de acuerdo sobre una clasificación de las industrias culturales. Desde el 2008, Naciones Unidas viene trabajando en la unificación de criterios, delimitación conceptual y aportación de datos estadísticos. Aun así, el Informe Economía Creativa 2013 recoge hasta seis modelos de clasificación de los sectores que abarca la economía creativa. Siguiendo la clasificación proporcionada por el IBGE (CNAE2), hemos seleccionado doce grupos de actividades, con el nivel de desagregación que proporciona la fuente para la escala local: confección de artículos de vestuario y accesorios (14); edición y edición de impresión (58); actividades cinematográficas, producción de vídeos y de programas de televisión, grabación de sonido y edición de música (59); actividades de radio y televisión (60); telecomunicaciones (61); actividades de servicios de tecnología e información (62); actividades de presentación de servicios de información (63); servicios de arquitectura e ingeniería, test y análisis de técnicas (71); actividades artísticas, creativas y de espectáculos (90); actividades relacionada con el patrimonio cultural y ambiental (91); actividades de exploración de juegos de azar y apuestas (92); y actividades deportivas, de recreo y ocio (93).

A pesar del desarrollo del concepto de economía creativa y de su repercusión en los ámbitos políticos y académicos, lo cierto es que la literatura sobre economía creativa, industrias creativas, clases creativas, o ciudades creativas han recibido numerosas críticas relacionadas tanto con su ambigüedad teórica y conceptual (PRATT; HUTTON, 2013), como por responder a los intereses de determinados grupos económicos, favorecer procesos de segregación y gentrificación urbana (PONZINI; ROSSI, 2010, BORÉN; YOUNG, 2013), pasando por ocultar procesos de especulación del suelo urbano (RODRÍGUEZ et al. 2005). 
Economía creativa, dinámicas locales y gobernaza en entornos metropolitanos: el caso de la región metropolitana de Salvador de Bahia

En primer lugar, en relación con su indefinición teórica y conceptual, varios autores han señalado el hecho de que el discurso sobre la economía creativa engloba una multiplicidad de sectores que en ocasiones no guardan relación entre sí, y difícilmente son amoldables a la idea de "clase" (PRATT; HUTTON, 2013). Además, se obvia la diversidad interna de muchas de las funciones de quienes trabajan en estos sectores, llegando incluso algunos estudios a abarcar empleados que no ejercen actividades creativas o relacionadas con la creatividad, sino que conllevan tareas rutinarias de apoyo a otros sectores, como puestos de trabajo relacionados con la logística o la reparación de equipamiento tecnológico, tal y como serían los empleados de las taquillas de los cines (NAVARRO et al., 2012) o de las industrias agroalimentarias. El resultado es una sobrevaloración del peso del sector cultural y creativo en la economía. A este respecto, Carava et al. (2013) señalan que en numerosos casos se sobrevalora la importancia de un conjunto de actividades cuyo peso relativo en la producción o el empleo totales hace discutible el valor estratégico que algunos les otorgan. A este respecto, una investigación de Calcagno y Cesín (2008), sobre el comercio exterior de bienes culturales en América del Sur, concluyó que el balance comercial de los productos culturales en Sudamérica tiene un déficit de 3.700 millones de dólares y que las importaciones totales son prácticamente el doble que las exportaciones. De acuerdo con el estudio, entre los siete países analizados (Argentina, Brasil, Colombia, Chile, Venezuela, Perú y Uruguay), sólo Brasil tiene un balance comercial positivo sobre los productos culturales. Si bien, tal y como indican los autores, estas exportaciones no son de productos característicamente culturales, sino que se trata de productos industriales como reproductores de DVDs, minicomponentes, televisores, etc.

En segundo lugar, numerosos autores critican que el modelo el desarrollo urbano asociado a las clases creativas ha resultado en procesos de gentrificación y segregación urbana. Andrew Harris (2011), analiza cuáles han sido los efectos de éstas en el distrito de Hoxton en Londres, donde los artistas repoblaron el panorama cultural de un espacio tradicionalmente de clase obrera en la década de los noventa, siguiendo la corriente de inversión inmobiliaria en la zona, pero también manipularon y menospreciaron la complejidad de las historias y las relaciones de clase y raza de los vecinos del barrio. El autor concluye que esta captura del espacio urbano a través de la clase creativa oculta nuevas formas de gentrificación de índole racista baseadas en el cambio de clase y raza de la población original. Por su parte, Lidia Mazo (2012), realiza un estudio comparado entre procesos similares en ciudades muy distintas: se analiza la cohabitación en el distrito chino de Milán y los cambios en Brooklyn para comprobar que una ciudad es siempre un espacio en constante mutación donde operan dinámicas de poder, haya o no un desplazamiento directo de la población.

En tercer lugar, Borén y Young (2013) critican que las políticas públicas vinculadas al desarrollo de la economía creativa responden en muchos casos a los imperativos económicos y a una agenda neoliberal. Así, ignoran los efectos negativos de la aplicación de estas políticas, relacionados con la inversión en grandes eventos culturales o deportivos para crear una suerte de ciudades "espectáculo" capaces de atraer turismo e inversión extranjera, en lugar de invertir en bienestar social, salud, educación o apoyo a otras formas de creatividad. En la misma línea, Vicario y Rodríguez (2005) sostienen que el proceso de de regeneración urbana experimentado por la ciudad de Bilbao durante los últimos quince años, y que han hecho del conocido "efecto Bilbao" un modelo de regeneración para otras ciudades, se resumen en "grandes operaciones urbanísticas e inmobiliarias para la renovación de "áreas de oportunidad", proyectos para la renovación y mejora del paisaje urbano, el uso del arte y la cultura como instrumentos para la regeneración de zonas y barrios degradados, campañas de

DRd - Desenvolvimento Regional em debate (ISSNe 2237-9029) 
promoción y marketing urbano, etc., que poco tienen que ver con la creatividad" (VICARIO; RODRÍGUEZ, 2005, p. 290).

Lo dicho hasta ahora no resta, sin embargo, para que la creatividad en sí misma sea en la actualidad uno de los motores clave para el desarrollo territorial y que determinados sectores "emergentes" empleen dicha creatividad en su funcionamiento. De hecho, el actual contexto de competitividad entre espacios ha demostrado beneficiar a aquellas ciudades que apuesten por desarrollar capacidades creativas diferenciadas (SCOTT, 2006, p. 13).

\section{ECONOMÍA CREATIVA Y GOBERNANZA URBANA}

El debate en torno al concepto de gobernanza urbana ha ido adquiriendo protagonismo en las últimas décadas y constituye un tema central en los debates sobre desarrollo urbano (MICHELINI, 2010). Aunque en Europa y Estados Unidos esas dinámicas se iniciaron en las décadas de los 70 y 80, en la mayor parte de los países de América Latina, se han intensificado durante la última década.

La crisis del sistema capitalista de la década de 1970 puso de manifiesto la inadecuación de los modelos teóricos tradicionales y supuso la aparición de un enfoque más local y transnacional de las políticas económicas (SANTOS, 2004). Los procesos de descentralización y re-escalamiento espacial asociados dejaron en manos de los gobiernos locales la búsqueda de soluciones para viejos y nuevos problemas- una vida urbana más compleja o la creciente polarización social y territorial, en un contexto de creciente competencia interurbana (MICHELINI, 2010). En este contexto, las instituciones públicas vieron crecer su agenda de intervención, pero aun así, el gran cambio de escenario dejó en entredicho las formas tradicionales, homogeneizadoras y segmentadas en que operan las políticas públicas (BAUMAN, 2003). Con el nuevo enfoque otorgado al desarrollo local, se produce un cabio en el papel que juega el Estado, dejando de ser el único con capacidad de decisión en los procesos de desarrollo (KLEIN, 2005). Se produce un giro en el marco teórico desde la perspectiva descendente a la perspectiva ascendente, buscando la participación de los actores locales en la toma de decisiones. Es decir, las políticas surgen desde el propio territorio, teniendo en cuenta las demandas y necesidades de las poblaciones locales y las características específicas de cada territorio (condiciones sociales, culturales e institucionales...). Dichas características están relacionadas con las relaciones sociales, la capacidad emprendedora de los actores, la formación de redes asociativas locales y la construcción de "capital social" (ALBURQUERQUE, 2005).

Es en este contexto donde se empieza a cuestionar la capacidad que tienen los gobiernos para dirigir el cambio social (crisis de gobernabilidad), proponiendo paradigmas alternativos de regulación del conflicto social que conllevan un retorno al mercado como única forma de regular las necesidades sociales (SUBIRATS, 2010). Dado que una de las transformaciones más radicales que experimentaron los espacios urbanos en ese período tiene que ver con la asunción de nuevas funciones por parte de los gobiernos locales y la transformación de sus estructuras político-institucionales hacia estructuras de gobierno más horizontales y participativas, una parte fundamental de los debates sobre desarrollo urbano relacionan la recuperación de las ciudades con su capacidad de emprendimiento, pero, sobre

DRd - Desenvolvimento Regional em debate (ISSNe 2237-9029) 
Economía creativa, dinámicas locales y gobernaza en entornos metropolitanos: el caso de la región metropolitana de Salvador de Bahia

todo, con la capacidad para construir coaliciones locales, con nuevos actores coherentes con nuevos objetivos de desarrollo. (MÉNDEZ, et al., 2006). Para ello, se recurre al estudio de nuevas formas de gobernar el territorio basadas en la noción de gobernanza, definida como un modelo alternativo de gestionar los asuntos públicos que implica la transformación y la recomposición de la acción pública, mediante la participación conjunta de los agentes institucionales, agentes privados y sociedad civil.

La gobernanza y la gobernabilidad son conceptos que ayudan a interpretar y analizar el grado de participación de la sociedad civil en una relación horizontal entre instituciones de gobierno con grupos vecinales, asociaciones civiles, organizaciones no gobernamentales, movimientos sociales y empresas privadas para dar cumpimiento de forma consensuada a visiones y requirimientos diferenciados (SOLÁ 2000). En palabras de R.A.W. Rhodes (1997, sacadao de SUBIRATS, 2010), el concepto de gobernanza viene a definir "[...] un nuevo proceso de goberno, una condición distinta de regulación social; un nuevo método a través del cual la sociedad es gobernada". También algunos autores, como Hirsch, identifican el gobierno en red con la innovación democrática: “[...] todas aquellas nuevas prácticas de coordinación a través de redes, partnerships y fórums deliberativos que nacen de las ruinas da representación centralizada, jerárquica y corporativa" (HIRSCH, 1972 sacadao de SUBIRATS, 2010).

Enfatizando la noción de territorio como una «construcción social y política» derivada de la acción colectiva de «grupos, intereses, e instituciones» (DAVOUDI, et al., 2008: 35), la noción de gobernanza urbana (ESPON, 2.3.2, 2006) pone el acento en los mecanismos de articulación de los actores para la construcción social de un proyecto de territorio (MICHELINI, 2010). La gobernanza, entendida como una nueva forma de regulación del conflicto social (SUBIRATS, 2010), y hacia formas "empresariales" de gobernanza urbana (HARDING, 1995, sacado de BLANCO, 2009), es un aspecto clave en la promoción de una economía creativa porque ciertas formas de gobernanza pueden, en contextos específicos, potenciar la creatividad y la innovación (HEALEY, 2004, sacado MICHELINI; MÉNDEZ, 2012).

No obstante, una revisión de la literatura sobre economía y ciudades creativas muestra que la consideración de estas articulaciones socio-institucionales posee particularidades que condicionan el éxito de los procesos de desarrollo local. Michelini y Méndez (2012) distinguen tres factores que intervienen en las estrategias de desarrollo. En primer lugar, las características de los actores implicados en el territorio. Por un lado, los regidores locales juegan un papel fundamental. Tal y como indican los autores, "[...] su orientación política, carácter más o menos emprendedor o características personales resultan determinantes tanto en las estrategias formuladas como en las coaliciones construidas" (MICHELINI; MÉNDEZ, p. 376, 2012). Los responsables locales están formando sus propios cauces para el desarrollo de la economía creativa al decidir dar su apoyo a este tipo de actividades y fortalecer las capacidades empresariales y de gestión de los actores culturales.

Relacionado con lo anterior, Foord, (2008) agrupa las intervenciones gubernamentales en las estrategias creativas en seis grandes categorías: i. Estrategias para proporcionar espacios de trabajo; ii. Formación y asesoramiento en el desarrollo de negocios; iii. Ayudas directas y préstamos a la creación de empresa; iv. Iniciativas fiscales; v. Eventos comerciales y tecnología; vi Infraestructura. Estas categorías no son excluyentes, sino que proporcionan un perfil de los principales tipos de intervención y, por tanto, los mecanismos utilizados para 
promover y apoyar a las empresas creativas en determinadas localidades. Muchas de las intervenciones son de apoyo al desarrollo económico común a las iniciativas de apoyo a las empresas genéricas - proporcionar espacio de trabajo y la formación, el asesoramiento, la creación de redes y los préstamos, capacidad de gestión empresarial y la educación creativa, el acceso a los eventos comerciales y nuevas tecnologías. Algunos son específicos para el sector creativo -incluyendo incentivos fiscales, como los impuestos turísticos y el utilizados en el aumento de la financiación para la inversión en infraestructuras artísticas en o créditos fiscales directos para los artistas creativos. Al igual que con los servicios de apoyo genéricos, muchas de las iniciativas de apoyo empresarial para el sector creativo se dirigen a empresas de nueva creación, mico o pequeñas y medianas empresas, y se manejan por agencias intermediarias del sector público o voluntario.

En esta línea, Borén y Young (2013) sostienen que mietras algunas formas de creatividad son valoradas por las élites urbanas y gozan de financiamento público-sobre todo aquellas que presentan una mayor visibilidad internacional-, otras menos "espectaculares" o de impacto internacional son ignoradas y excluídas de las políticas públicas encaminas al desarrollo de las ciudades creativas.

En el otro extremo, Michelini y Méndez (2012) señalan que las contrariedades de la política partidaria, la búsqueda de un retorno electoral inmediato o la falta de una política municipal consistente pueden frenar el desarrollo de la economía creativa. Los demás actores juegan también un papel clave en los procesos de elaboración de políticas y estrategias para impulsar la economía creativa.

Un segundo aspecto de interés tiene que ver con las culturas de gobernanza que están detrás de los procesos de negociación, es decir, las normas, valores, costumbres y convenciones que configuran el ámbito de la acción colectiva de una comunidad, haciendo que el debate sea más o menos renovador. A su vez, el Informe UNTACD (2013) identifica otros factores fundamentales de éxito que van desde el desarrollo de las capacidades locales hasta la gestión propia de los bienes por parte de los habitantes, pasando por la facilitación de las conexiones y los flujos transnacionales.

Por último, Michelini y Méndez (2012) llaman la atención sobre la necesidad de entender la Administración como una realidad compleja con una multiplicidad de ámbitos de intervención, con reglas y dinámicas de actuación muy diversas, es decir, una forma de poligobernanza.

\section{¿ES LA ECONOMÍA CREATIVA EN SALVADOR DE BAHÍA UN VECTOR DE DESARROLLO?}

La economía creativa es reconocida hoy en día en Brasil como uno de los ejes transformadores del proceso de cambio que está teniendo lugar en la potencia emergente (SIERRA, 2015). El impulso a la economía creativa empezó en 2004, coincidiendo con la organización en la ciudad de Sao Paulo de la undécima sesión de la Conferencia de las Naciones Unidas sobre Comercio y Desarrollo (UNCTAD XI), previa al Foro Internacional sobre el Desarrollo de un Centro Internacional de Industrias Creativas en Salvador de Bahía,

DRd - Desenvolvimento Regional em debate (ISSNe 2237-9029) 
en 2005. Paralelamente, el gobierno de Lula da Silva (2003-2010) supuso un cambio en la articulación de las políticas públicas pasando de un de un enfoque neoliberal a una visión de la política cultural de Estado basada en la descentralización, la participación y articulación interna (SIERRA, 2015). En este nuevo marco político de gobernanza, el punto álgido en el proceso tuvo lugar en 2012 con la creación de la Secretaría de Economía Creativa, fundada a partir de cuatro principios rectores: educación, competencias creativas, infraestructura para la cadena de valor de bienes y servicios creativos, y la creación/ajuste de un marco legal para los sectores creativos. Con una clara intención de privilegiar el ámbito local como eje articulador de le economía creativa, la Secretaría de Economía Creativa tiene como reto alcanzar el $60 \%$ de las ciudades del país, prueba de la magnitud y relevancia del sector creativo en las políticas públicas brasileñas (SIERRA, 2015).

Desde el año 2004 se instauraron una serie de medidas legislativas y programas de apoyo al sector creativo, como Río Creativo (2010), que tiene como uno de sus pilares fundamentales la creación de dos "incubadoras" de emprendedores creativos, y que conllevó la selección en 2011 de 21 proyectos para recibir formación y asesoramiento de gestión y técnica. Paralelamente, el Servicio Brasileño de Apoyo a las Pequeñas y Medianas Empresas (SEBRAE), la institución privada más activa en la promoción de la economía creativa, lleva mucho tiempo trabajando en varios campos creativos, como la artesanía, el turismo cultural y la industria musical El SEBRAE de Bahía ha organizado recientemente una serie de 10 conferencias dirigidas mayoritariamente a los nuevos administradores que han sido nombrados en la ciudad. Más de 100 ciudades participan ahora en esta iniciativa y se discuten futuras acciones entre el sector privado y el Gobierno del estado de Bahía. Otra iniciativa fue la creación de la "Red de Agentes Públicos de la Economía Creativa", conformada por representantes de las secretarías de cultura de 18 estados brasileños para diseñar e implementar políticas integradas que fomenten la economía creativa.

El interés del gobierno brasileño para impulsar el sector creativo en las esferas federal, municipal y estadual queda patente en el aumento del total de inversiones gubernamentales en el apoyo a la cultura, pasando de 4,4 billones en 2007 a aproximadamente 7,3 en 2010. Según Sierra (2015), las buenas previsiones de crecimiento del sector en el PIB, hasta del 4,5\%, marcan la voluntad política concreta de convertir la economía de la cultura en un polo de desarrollo y vector de nuevos procesos de crecimiento y bienestar social, sin parangón no solo en la historia del país sino en la propia geopolítica regional. Sin embargo, tal y como señala el autor, la actual política pública plantea retos de articulación intergubernamentales, políticoadministrativos y socioculturales problemáticos para Brasil.

A pesar de la importancia dada hoy en día a la economía creativa en Brasil como vector de desarrollo, existe una carencia de análisis del sector cultural en su dimensión económica. Por ejemplo, según el Plano de la Secretaría de Economía Creativa, la contribución de los sectores creativos al PIB brasileño en el año 2010 representaba, en valores absoluto, 104,37 billones de reales, lo que representa un 2,84\% del PIB. Si bien la contribución del sector creativo a la economía es residual, es importante destacar que el crecimiento anual de los últimos 5 años del núcleo de los sectores creativos $(6,13 \%)$ fue superior al crecimiento medio anual del PIB brasileño (4,3\%).

Dentro del país, Bahía destaca por ser un estado representativo de la producción cultural brasileña, lo que justificó, en parte, la propuesta de implantación del Centro Internacional Transdisciplinar de Economia Creativa en la ciudad de Salvador. En período 
2007-2010, el Estado de Bahía presentó un incremento en valores absolutos de 126,63 millones de reales a 203,73 millones (BAHIA CRIATIVA, 2014). Además, un informe sobre el empleo en el sector realizado por el gobierno del estado de Bahía (BAHIA CRIATIVA, 2014), con datos del IBGE y de la PNAD de 2012, reveló que el sector cutural/creativo ocupa a un número de 3.6 millones de personas en todo el país, estando al Región Nordeste en cuarta posición dentro de las grandes regiones, del total de los ocupados en el segmento creativo. A nivel de las capitales de región, Bahía posee 171 mil ocupados en el sector creativo, lo que representa un 2.6\% del total de ocupados en el estado. De estos, 65 mil trabajan por cuenta propia, 46 mil son empleados con cartera firmada, 42 mil son empleados sin cartera firmada y 10 mil son empresarios. En cuanto al rendimiento de la población ocupada, el informe revela que el rendimiento medio de esa población es de 1.553 reales, valor ligeramente superior al rendimiento medio de la población en los demás sectores de la actividad productiva (en torno a 1.460 reales). El estado de Bahía figura como el tercer estado de mayor rendimiento medio por ocupados, detrás de Río de Janeiro y Sao Paulo.

Un estudio de Castro Lima (2006) identifica algunas características del empleo en las actividades creativas en el estado de Bahía. En primer lugar, se trata de un segmento predominantemente masculino y con una mayor representación de personas de color blanca. Además, poseen un nivel de formación elevado en relación a la media de la población ocupada total, y representan una mayor proporción porcentual en el segmento de trabajadores autónomos. Por último, el estudio demuestra que el núcleo de las industrias creativas tiene una participación mayor en relación a la población ocupada total, que segmentos importantes de la economía bahiana, como la industria química, el cultivo de soja, papel y celulosa y la industria textil y vestuario. Al igual que en otros casos analizados, el estudio contiene limitaciones importantes en relación al tratamiento estadístico debido a la dificultad de desagregación de los datos.

\section{MAPA DE LA ECONOMÍA CREATIVA EN LA REGIÓN METROPOLITANA DE SALVADOR}

La RMS ocupa una posición destacada en el escenario metropolitano brasileño. Según datos del Observatório das Metrópoles (2014), la RMS ocupa la octava posición en población, en Producto Interno Bruto y en Renta Per Cápita, y posee una fuerte base industrial y de servicios, aunque en términos de gestión empresarial no destaca en el contexto nacional. A pesar de ser una de las regiones más importantes del Nordeste y Norte brasileño, sus indicadores sociales la sitúan muy por debajo de las metrópolis y regiones metropolitanas del Sur y Sudeste.

A nivel turístico, las inversiones gubernamentales en infraestructuras y la inversión privada en hoteles, resorts y otros equipamientos, consiguieron una importante expansión del sector. Los principales atractivos de la región están asociados a las bellezas paisajistas, la historia y la cultura popular bahiana. El carnaval de Salvador posee una fuerte repercusión nacional e internacional, atrayendo cada año a miles de turistas. A nivel nacional, Bahia posee el mayor número de grandes resorts de Brasil, la mayoría de ellos localizados en el Litoral Norte, bajo la influencia de la metrópoli.

DRd - Desenvolvimento Regional em debate (ISSNe 2237-9029) 
Economía creativa, dinámicas locales y gobernaza en entornos metropolitanos: el caso de la región metropolitana de Salvador de Bahia

La estructura interna de la RMS muestra un fuerte desequilibrio en la relación tamañojerarquía, observándose una fuerte macrocefalia de la capital, Salvador, tanto en términos demográficos como, y sobre todo, económicos. Como consecuencia, las desigualdades y los problemas intrametropolitanos son bastante expresivos.

Según Moreira y Corso, (2014), tanto la capital bahiana, como otros municipios de la RMS, vienen experimentando ciertos cambios y tendencias comunes a otras ciudades brasileñas y latinoamericanas. Estos cambios se resumen en: la expansión hacia las áreas periurbanas; el abandono, deterioro o gentrificación de antiguas áreas centrales; la edificación de equipamientos de gran impacto en la estructuración del espacio urbano, como shoopings centers, centros de convenciones, etc; la difusión de nuevos patrones habitacionales e inversiones inmobiliarias destinadas a los grupos de media y alta renta, provocando una suerte de autosegregación de las rentas altas; $y$, finalmente, la afirmación creciente de la lógica del capital, con el abandono del Estado de buena parte de sus funciones tradicionales de planeamiento y gestión urbana y su transferencia para actores privados. Para los autores, estos patrones son resultado de un nuevo enfoque de gobernanza que sustituye el modelo racionalista y funcionalista por un modelo de "emprendimiento urbano". Esa gobernanza se inspira en conceptos y técnicas del mundo empresarial convirtiendo a la ciudad en un agente económico, y sitúa como eje central de la cuestión urbana, la búsqueda de una competitividad orientada a la atracción de capitales. El resultado, es una mercantilización y "espectacularización" de la ciudad, con la edificación de grandes equipamientos culturales, la gentrificación de ciertas áreas, la atracción de grandes eventos internacionales, el establecimiento de asociaciones público-privadas y una mayor flexibilidad y libertad para las operaciones financieras. Como resultado del cambio en la orientación de la gestión y el planeamiento de las áreas urbanas, Moreira y Corso (2014) concluyen que se están favoreciendo especialmente las inversiones inmobiliarias y la especulación del suelo urbano.

La ciudad de Salvador, primera capital de Brasil, también experimentó cambios importantes en las últimas décadas en su configuración espacial. La construcción civil y las actividades inmobiliarias experimentaron un fuerte crecimiento en las últimas décadas. Según Freitas et al. (2006), Salvador se transformó en una ciudad policéntrica con tres centros formalizados en su Plano Director de Desarrollo Urbano (PDDU). El primer centro de la ciudad, el Centro Tradicional, data del siglo XVI. El segundo, el Centro do Camaragibe (también conocido como centro de Iguatemi), se urbanizó en la década de 1970. El tercer centro, el Centro do Retiro- Acesso Norte, aún está en fase de formación. No obstante, a pesar de la existencia de esas tres centralidades previstas en el PDDU, el área central de la ciudad de Salvador, el Centro Tradicional, todavía conserva un papel central y concentrador de las actividades urbanas. Sin embargo, la ciudad de salvador posee la paradoja de poseer un Centro Tradicional poco accesible para los ciudadanos en contraposición a los centros económicos y de consumo, creados a través de intereses públicos y privados con el objetivo de modernizar la ciudad (SANTOS, ANDRADE y FREITAS, 2010).

Más allá de estos tres centros formalizados en el PDDU, en la ciudad de Salvador eclosionaron una multitud de centros y subcentros que, además, son espacios muy diferenciados entre sí, en cuanto a sus características sociales y económicas. En este sentido, las condiciones de pago del suelo urbano, creados por los nuevos intereses financieros e inmobiliarios generó nuevas desigualdades de acceso a la ciudad, materializándose en nuevas formas de segregación espacial (SANTOS, 2010). El aumento de la población en los últimos años agravó el proceso de expansión periférica del territorio municipal siguiendo patrones de

DRd - Desenvolvimento Regional em debate (ISSNe 2237-9029) 
uso y ocupación del suelo de baja calidad urbana (FREITAS et al., 2006). La formación de los nuevos centros no es siempre una demanda de la mayoría de los moradores de la ciudad, sino una respuesta a las demandas de ampliación de capital (OLIVEIRA, 2011). Esto revela conflictos e intereses en la ciudad que tienen sus efectos en el proceso de producción y reproducción del espacio urbano (SANTOS, 2010). En este sentido, nos parece fundamental conocer en papel de las industrias creativas en este nuevo proceso de segregación espacial.

Para analizar la contribución de las industrias y servicios culturales y creativos en la RMS, hemos estudiado la distribución espacial del empleo. Para el estudio, no hemos podido contabilizar el total de municipios que conforman el área metropolitana, pues el IBGE solo proporciona datos de aquellos municipios de más de 50 mil habitantes, quedando fuera del estudios los municipios de Itarica, madre de Deus, Mata de São Jao, Pojuca, São Francisco do Conde, São Sebastião do Passé y Vera Cruz, que juntos representan el 6\% de la población de la región metropolitana.

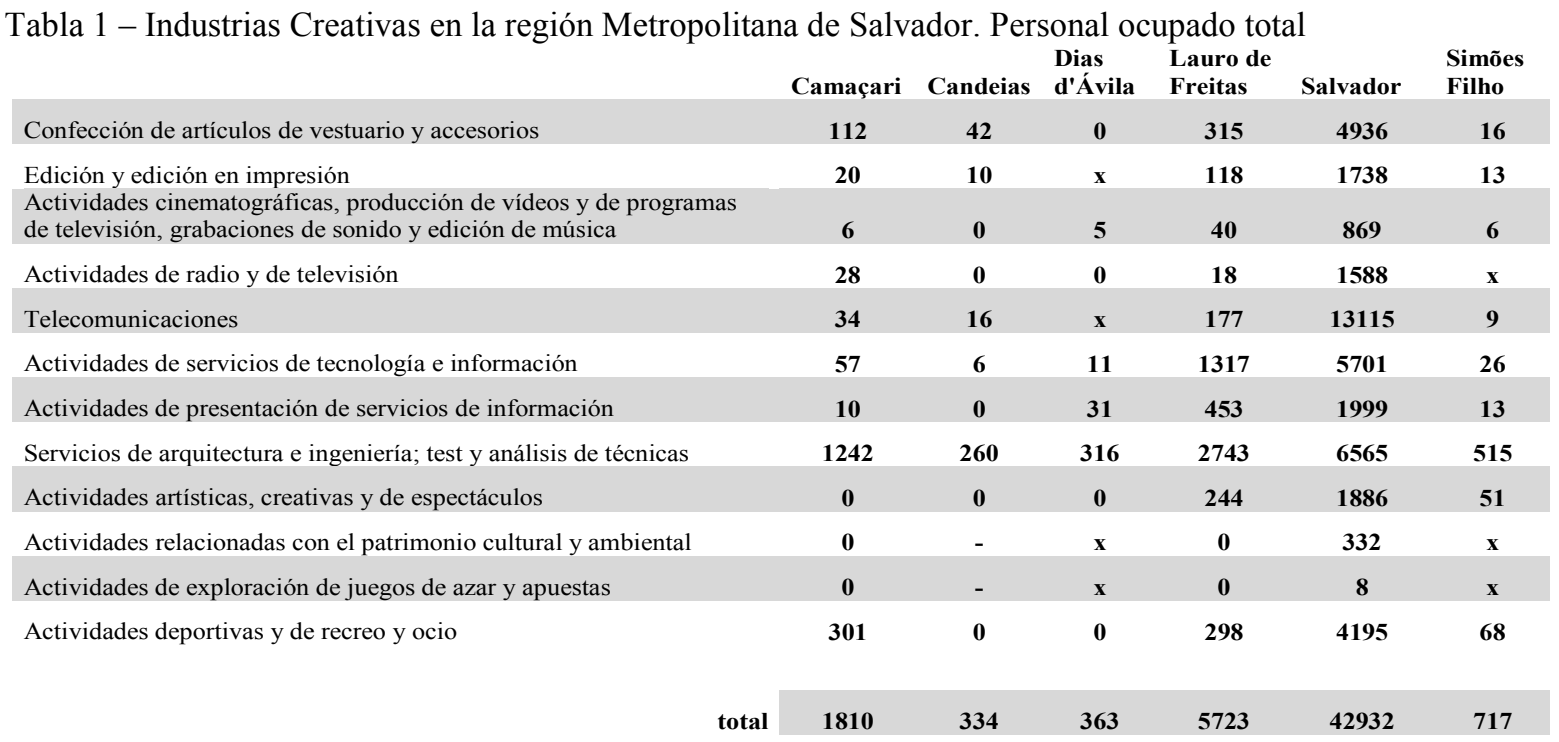

Fuente: elaboración propia a partir de IBGE, 2015

Una primera visión de las industrias culturales y creativas en le región, muestra una clara preponderancia de la capital, Salvador, por encima del resto de municipios, algo previsible pues la capital concentra el 74\% de la población de la RMS. El municipio Salvador contaba a finales de 2012 con 156.305 afiliados en la economía creativa, que vienen a representar un $6 \%$ del total del empleo.

La distribución sectorial de actividades en la región metropolitana muestra contrastes importantes, confirmándose el sesgo hacia algunos servicios avanzados y actividades profesionales en general, como servicios de telecomunicaciones, tecnologías de información y arquitectura e ingeniería; frente a esto se advierte un peso relativo menos importante de las actividades ligadas con el patrimonio y la cultura.

Es importante destacar que este estudio no tiene en cuenta aquellas actividades englobadas en la economía sumergida. Este datos es importante pues se estima que el número sea muy elevado en la RMS: pero además, su peso es muy destacado en el grueso de las actividades artísticas, creativas y culturales, lo cual podría hacer mudar sustantivamente el mapa de trabajadores de la economía cultural y creativa (SERPA, 2007). 
A escala metropolitana, la economía creativa sigue mostrando una elevada macrocefalia en la ciudad de Salvador, contribuyendo poco a la construcción del modelo territorial policéntrico que se viene buscando en la ciudad en las últimas décadas. No obstante, se observa una presencia importante de los servicios de arquitectura e ingeniería y tecnologías de la información en otros municipios metropolitanos como Lauro de Freitas o Camaçari.

En este sentido, los miembros de la clase creativa se ubican en determinadas ciudades y regiones y en ellas no se distribuyen de forma homogénea (FLORIDA, 2002; FOORD, 2008; CLIFTON; COOKE, 2009; LAZARETTI et al. 2008, MÉNDEZ; SÁNCHEZ, 2010; MICHELIN; MÉNDEZ, 2012). Tal y como indica Foord (2008), las profesiones más tecnológicas y de orientación económica prefieren residir en mayor medida en los núcleos urbanos de las áreas metropolitanas, mientras que las ocupaciones más relacionadas con la cultura suelen elegir los centros históricos de las ciudades, con una escena cultural y social vibrante (MOMMAAS, 2004). Estas características también se dan en la RMS, siendo el centro histórico del Pelourinho núcleo de importantes manifestaciones culturales y artísticas.

Ello parece demostrar que sigue siendo importante la proximidad física para determinados tipos de actividades, mientras algunas interpretaciones valoran la concentración de estas actividades al entender que refuerzan los atractivos urbanos, por otra, se critican los posibles efectos de segmentación urbana que pueden producir.

La observación de los datos indica que la economía creativa se muestra poco sensible a las tendencias difusoras que afectan a otras actividades en las últimas décadas, como el empleo en la industria de transformación (BANDEIRA et al. 2014), reforzando con ello el papel centralizador del núcleo de Salvador. A esta macrocefalia de Salvador, que se da tanto en relación al Estado como a la región metropolitana, se une la falta de articulación de un sistema de gobernanza en la región. Según un estudio de Franco, Baggi y Ferreira (2013, citado por BANDEIRA et al., 2014), la RMS no posee dotación presupuestaría para el Fundo Metropolitano desde 2008, el Conselho Consultivo e Deliberativo es inoperante, y no existe un órgano metropolitano ni un sistema de gestión, confirmando la fragilidad institucional de la región. Como resultado, las desigualdades y los problemas intrametropolitanas son importantes. 
Figura 1 - Distribución del empleo en la Economía Creativa en la RMS

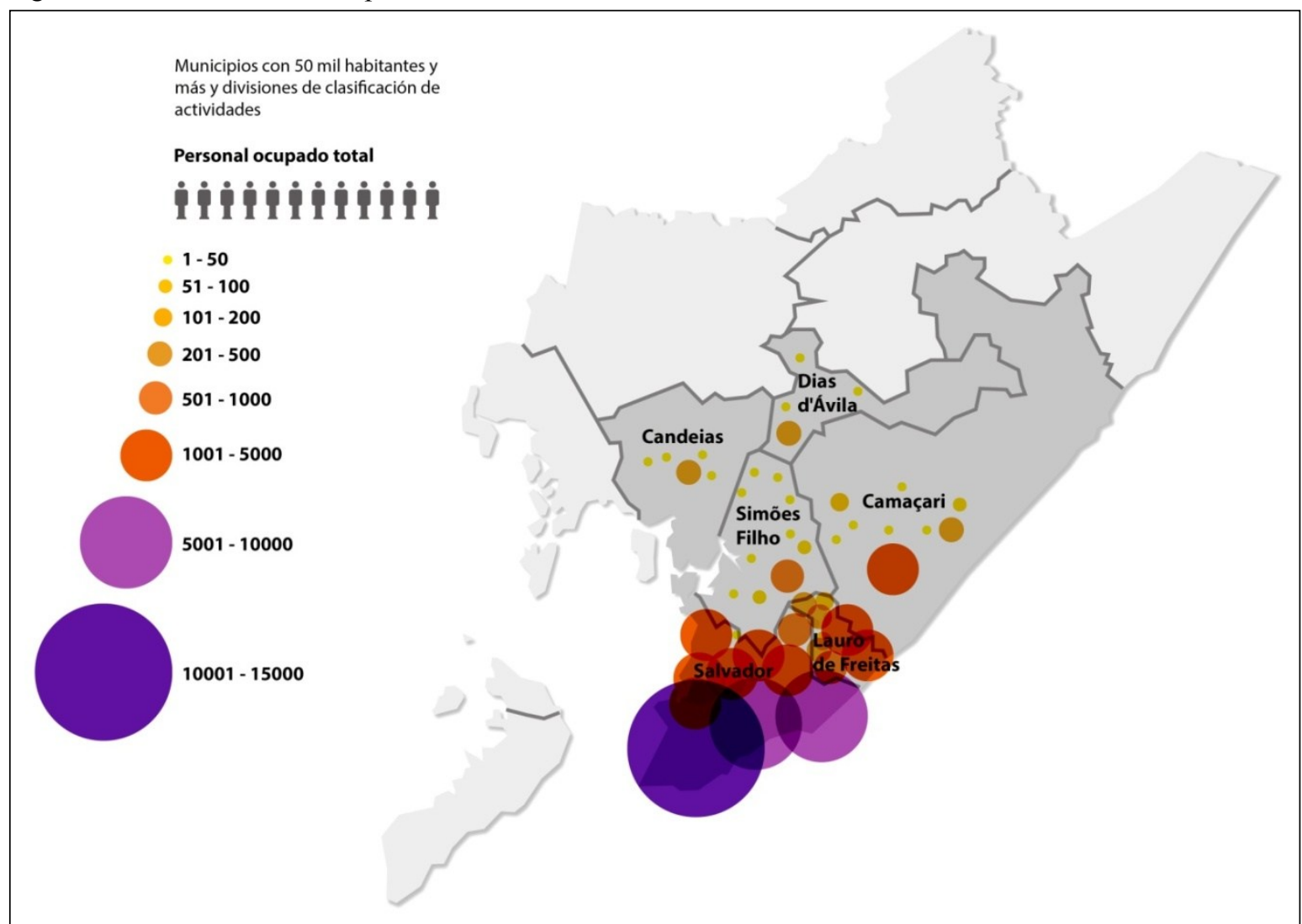

Fuente: elaboración propia, 2015

Como resultado, la selectividad territorial, no sólo de las industrias, sino también de la clase creativa podría generar nuevas formas de segmentación territorial y metropolitana. Por tanto, es necesario desarrollar un proceso profundo de planeamiento y de gobernanza territorial metropolitano que englobe proyectos sectoriales que actualmente se están gestionando de manera aislada. La solución, según Bandeira et al. (2014), pasa por construir una estrategia de desarrollo metropolitano a medio plazo que integre al Estado de Bahia, la Unión, municipios, empresas y sociedad organizada con la participación de las universidades.

Lo dicho hasta ahora no resta para que la economía creativa sea reconocida como una estrategia clave en el desarrollo urbano. Como se ha venido señalando, es necesario incluir en el análisis de la economía creativa otros aspectos vinculados a cuestiones sociales y no solamente enfocadas al beneficio económico (PRADA, 2015). En este sentido, la economía creativa también puede favorecer la dinamización de centros urbanos que actualmente se encuentran en una situación de declive poblacional y funcional. La reconversión de antiguas áreas o puertos industriales en polos de innovación, dotando a esas áreas de la infraestructura necesaria para las actividades creativas es una herramienta de gestión importante para revitalizar dichas áreas, siendo un buen ejemplo de ello 22@ de Barcelona. Asimismo, en la esfera de la iniciativa ciudadana están surgiendo actividades de inserción social, reivindicación, solidaridad, etc, que buscan acercarse a las actividades económicas desde la creatividad, la innovación y el arte como modo de subsistencia de grupos excluidos, dando lugar a una "reconciliación" entre la creatividad y el desarrollo urbano desde un punto de vista 
socialmente sostenible. La existencia de las trayectorias locales y las redes locales de actores son elementos claves de su éxito (PRADA, 2015)

El proyecto Territorios Creativos impulsado en la ciudad de Salvador de Bahia, actúa en este sentido apostando por la creatividad del emprendimiento local, a través de los llamados siete territorios creativos: Candeal, Rio Vermelho, Ribeira, Bonfim, Comércio, Santo Antônio Além do Carmo y Curuzu. El proyecto, surgido en 2011 con el objetivo de incentivar la competitividad de los destinos turísticos de Salvador. En 2013, el proyecto pasó a formar parte del Proyecto Estructural de la Economía Creativa de Bahía, recibiendo el apoyo del Sebrae Nacional, con la disposición de fomentar los pequeños negocios en eses siete territorios creativos. La marca de identidad, los saberes familiares y tradicionales, la cultura y la historia, la gastronomía, la autenticidad cultural y productiva, o el arte, son algunas de las ideas desarrolladas en negocios empresariales. El proyecto desenvuelve acciones innovadoras en el segmento creativo, buscando siempre una lógica de actuación participativa, defendiendo la valorización de las comunidades, la promoción de la calidad de vida y la transformación socioeconómica.

En este y otros proyectos similares de regeneración, los jóvenes asumen un papel relevante en la puesta en marchas de actividades que no requieren infraestructuras de gran dimensión en el desarrollo de productos creativos. Estos jóvenes inician proyectos en espacios muchas veces degradados o con pocas condiciones, con un coste menor, permitiendo adaptarlos a sus necesidades.

En definitiva, la economía creativa se trata de un importante medio para revitalizar las áreas centrales que tienden a despoblarse y, al mismo tiempo, fijar población. Sin embargo, estas iniciativas también pueden generar segregación o la expulsión de los residentes. Tal es el caso del popular barrio del Pelourinho, en el Centro Tradicional de la Ciudad, convertido actualmente en unos de los centros culturales y turísticos más importantes de la ciudad, pero que conllevó la expulsión de los residentes de sus viviendas, y la ocupación de los nuevos espacios por actividades de comercio y servicios, para su consumo en forma de entretenimiento dirigidas a la clase media local e incremento del flujo turístico (DUARTE, 2000).

\section{CONSIDERACIONES FINALES}

La Región Metropolitana de Salvador viene destacando por su amplia producción cultural Muestra de su importancia internacional, fue la definición de implantar el Centro Internacional Transdisciplinar de Economía Creativa en la ciudad de Salvador.

El presente artículo ha tratado de explorar la dinámica de la economía creativa en la RMS. Para ello, hemos utilizado el modelo realizado previamente por Michelini y Méndez (2012) en la Región Metropolitana de Madrid. Sin embargo, no fue posible realizar un tratamiento estadístico adecuado debido a las limitaciones de las fuentes de datos disponibles. A pesar de esas limitaciones, fue posible identificar algunas características interesantes del conjunto de las actividades creativas en la región. 
Por un lado, las estadísticas sobre economía creativa ponen de manifiesto el crecimiento de este tipo de actividades en el mundo. No obstante, el peso de estas actividades en la economía es discutible y tiende a sobrevalorarse. Un análisis más detallado muestra que este crecimiento tiene un carácter eminentemente local favoreciendo la definición de una nueva jerarquía metropolitana (MICHELINI; MÉNDEZ, 2014).

Las economías creativas tienden a la concentración debido a las externalidades positivas que genera, aunque en una escala local la herencia cultural, el patrimonio inmaterial disponible y las políticas públicas implementadas pueden resultar determinantes en el desarrollo de los distritos culturales y clusters. La distribución espacial de las industrias y servicios creativos en la Región Metropolitana de Salvador contribuye muy modestamente a la configuración de estructuras territoriales policéntricas. No obstante, los clusters creativos no son agrupaciones empresariales convencionales y se ponen en evidencia que otros factores son fundamentales para su desarrollo y forma (especialmente el papel de las artes con fondos públicos e instituciones culturales). Los objetivos estratégicos de los clusters creativos son diferentes de las agrupaciones empresariales convencionales y, en muchas ocasiones, tiene como objetivo favorecer la inclusión social, el desarrollo cultural y el emprendimiento.

En este sentido, el caso de la región metropolitana de Salvador de Bahía también rompe con la idea de ventaja de deslocalización de las empresas convencionales, pues la localización geográfica de las empresas del sector cultural y creativo está relacionada con factores como la historia, las experiencias culturales y heredadas de cada territorio. Estos factores dificultan la imitación de modelos de éxito desarrollados en otros territorios puesto que las particularidades de cada territorio pueden hacer fracasar proyectos de intervención pública o no proporcionar los resultados esperados. La frecuente tentación de adoptar de forma acrítica determinados modelos, estrategias o recetas de desarrollo surgidos en otros contextos exige, pues, mantener en este caso una perspectiva crítica en relación con el papel de las industrias y servicios creativos como factor de desarrollo urbano (MICHELINI; MÉNDEZ, 2014). Tal y como indica Foord (2008), no todas las localidades pueden convertirse en territorios creativos con las ventajas competitivas que ello implica.

También es necesaria una reevaluación de las expectativas, a veces poco realistas, respecto a la expansión de la economía creativa. Es innegable que existen evidencias sobre su importancia y significado en los estratos superiores del sistema urbano y en ciudades que han puesto en valor sus recursos culturales heredados o han sido capaces de generar otros. Sin embargo, la crisis europea y la caída del empleo en el sector creativo en países como Reino Unido, ponen en evidencia la necesidad de profundizar sobre las necesidades y las prácticas de este sector y su relación con otros sectores en la economía del conocimiento.

Asimismo, las actividades y servicios creativos pueden convertirse en herramientas de revitalización socioeconómica de ciertas áreas en declive y combatir la tendencia de abandono de ciertos centros urbanos, por lo que deberían ser tenidas en cuenta en la elaboración de las políticas urbanas. Es evidente, pues, que continúa siendo un importante reto para la RMS potenciar este tipo de actividades. El éxito estará muy relacionado con las trayectorias locales y la capacidad de conformar redes de cooperación socio-institucional, estrechamente asociadas a la gobernanza (CARAVACA et al., 2013).

Finalmente, se sugiere combinar estudios cuantitativos sobre el peso y la distribución de la economía creativa en contextos específicos con estudios cualitativos sobre iniciativas no 
tan volcadas al beneficio económico como a la búsqueda de un desarrollo más sostenible y solidario, que permitan una mayor compresión del significado de la economía creativa.

\section{REFERENCIAS}

ALBURQUERQUE, F. Reflexiones sobre el desarrollo económico en la práctica: desarrollo y territorio", CSIC, Madrid, noviembre de 2005

AUSTRALIA. Creative nation: Commonwealth cultural policy, Department of Communications and the Arts. Octubre, 1994.

BAUMAN, Z. Comunidad. En busca de seguridad en un mundo hostil, Siglo XXI, Madrid, 2003

\section{BAHIA CRIATIVA. DIRETRIZES PARA O DESENVOLVIMENTO DA ECONOMIA}

CRIATIVA NA BAHIA. Secretaria de Cultura do Estado da Bahia, 2014

BANDEIRA, S.; NENTWIG; PIRAJÁ. Salvador: transformações na ordem urbana [recurso eletrônico]: metrópoles: território, coesão social e governança democrática. / organização Inaiá Maria Moreira de Carvalho, Gilberto Corso Pereira ; coordenação Luiz Cesar de Queiroz Ribeiro. - 1. ed. - Rio de Janeiro : Letra Capital : Observatório das Metrópoles, 2014.

BLANCO, I. Gobernanza urbana y políticas de regeneración: el caso de Barcelona. Revista española de ciencia política, n. 20, p. 125-146, 2009.

BORÉN, T., y YOUNG, C.Getting Creative with the 'Creative City'? Towards New Perspectives on Creativity in Urban Policy. International Journal and Regional Research, v. 37, n. 5, p. 1799-1815, 2013.

BRASIL. Plano da Secretaria da Economia Criativa: políticas, diretrizes e ações. 2011 2014. Brasília: Ministério da Cultura, 2011.

CALCAGNO, N. y CESIN, E. E. Nosotros y los otros. El comercio exterior de bienes culturales en América del Sur, 1.ed. Buenos Aires: Secretaría de Cultura de la Presidencia de la Nación, 2008.

CARAVACA, I; GONZÁLEZ, G.; FENANDEZ, V. Y GARCÍA, A. Economía creativa en la aglomeración metropolitana de Sevilla: agentes, redes locales de colaboración y principales actuaciones. Boletín de la Asociación de Geógrafos Españoles, n. 63, p. 81-104, 2013.

CASTRO L. Debate sobre indústrias criativas: uma primeira aproximação para o Estado da Bahia. Revista Desenbahia, v.3, n. 5, set. 2006.

CLIFTON, N. Y COOKE, P. Creative knowledge workers and location in Europe and North America: a comparative review. Creative Industries Journal, v. 2, n. 1, p. 73-89, 2009. 
DAVOUDI, S.; EVANS, N.; GOVERNA, F. Y SANTANGELO, M. Territorial governance in the making. Approaches, methodologies, practices. Boletín de la AGE, n. 46, p. 33-52, 2008 .

DUARTE. Innovación y renovación urbana. El caso del centro histórico de Salvador-Bahia en Brasil. Scripta Nova. Revista Electrónica de Geografía y Ciencias Sociales. Universidad de Barcelona, 1 ago. 2000.

ESPON 2.4.2 PROJECT. Integrated Analysis of Transnational and National Territories Based on ESPON Results - Draft Final Report, 2006.

FLORIDA, R. The rise of the creative class, Nueva York, Basic Books, 2002.

FONSECA A.C. La Agenda pendiente de la economía creativa en Brasil - eppur si muove. Periférica: Revista para el análisis de la cultura y el territorio, n. 15, p. 247-257, 2014. (Ejemplar dedicado a: Periférica Internacional).

FOORD, J. Strategies for creative industries: an international review. Creative Industries Journal, v. 1, n. 2, p. 91-113, 2008.

FRANCO, C. R.; BAGGI, M. S.; FERREIRA, M. G. Governança na Região Metropolitana de Salvador: como estamos? COSTA, Marco Aurélio; TSUKUMO, Isadora Tami Lemos (orgs.). 40 anos de Regiões Metropolitanas no Brasil. Brasília: IPEA, p. 187-203, 2013.

FREITAS, P.A. Y NASCIMENTO, M. X. (2006): Comportamento espacial e estrutural da indústria baiana no período 1994-2003. Revista Desenbahia, n. 5, p. 7-30, 2006.

GARRIDO, I.M. Modelos multiorganizacionais no turismo: Cadeias, Clusters e Redes.

Salvador: Secretária da Cultura e Turismo, 2002, 152, p. 1-(Colección Selo e Trusimo).

HARDING, A. Elite Theory and Growth Machines. In: David Judge, Gerry Stoker y Harold Wolman, eds. Theories of Urban Politics. Londres: Sage, 1995.

HARRIS, A. Art and gentrification: Pursuing the urban pastoral in Hoxton, London. Cities, n. 33, p. 29-35, 2011.

HEALEY, P. Creativity and urban governance. Policy Studies, n. 25, p. 87-102, 2004.

HOWKINS, J. The Creative Economy: How People Make Money from Ideas. London: Penguin Books, 2001.

KLEIN, J.L. Innovación social y gobernabilidad plural en la reconversión metropolitana de Montreal ¿Excepción o modelo? Colección Études théoriques. CRISES. Montreal, 2004.

LAZZERETTI, L.; BOIX, R.; CAPONE, F. Do creative industries cluster? MappingvCreative Local Production Systems in Italy and Spain. Universitat Autònoma de Barcelona, 2008. Departamento de Economía Aplicada. Disponible en http://www.ecap.uab.es/RePEc/doc/wpdea0805.pdf. Consultado en 10 abr. 2014.

MANZO, L. On People In Changing Neighborhoods. Gentrification and Social Mix: Boundaries and Resistance. A comparative ethnography of two historic neighborhoods in

DRd - Desenvolvimento Regional em debate (ISSNe 2237-9029) 
Milan (Italy) and Brooklyn (New York, USA), Cidades, Comunidades e Territórios, n. 24, p. 1-29, 2012.

MÉNDEZ, R., MICHELINI, J.J., ROMEIRO, P. Redes socio-institucionales e innovación para el desarrollo de las ciudades intermedias. Ciudad y Territorio, v. 38, n. 148, p. 377-395, 2006.

MÉNDEZ, R; SÁNCHEZ, S. Spanish cities in the knowledge economy: Theoretical debates and empirical evidence. European Urban and Regional Studies, v. 18, n. 2, p. 136-155, 2010 .

MÉNDEZ, R; MICHELINI, J.J.; PRADA, J. Y TÉBAR, J. Economía creativa y desarrollo urbano en España: una aproximación a sus lógicas espaciales. EURE: Revista Latinoamericana de Estudios Urbano Regionales, n. 113, p. 5-32, 2012.

MICHELINI, J.J.; MÉNDEZ, R. Economía creativa y estrategias de promoción en la Región Metropolitana de Madrid. Crisis económica e impactos territoriales - V Jornadas de Geografía Económica AGE Univ. de Girona, 2012.

MICHELINI, J.J. Gobernanza territorial, iniciativas locales y desarrollo urbano: los casos de Getafe y Alcázar de San Juan. Boletín de la Asociación de Geógrafos Españoles, n.. 54, p. 175-201, 2010.

MICHELINI, JJ. Y MÉNDEZ, R. La economía creativa en la crisis: contrastes espaciales y estrategias de promoción en la región metropolitana de Madrid. In: PUEBLA, Juan Miguel Albertos; SÁNCHEZ HERNÁNDEZ, José Luis (Coords.) Geografía de la crisis económica en España, 2014. p. 667-690

MOMMAAS, H. "Cultural clusters and the post-industrial city: towards the remapping of urban cultural policy”. Urban Studies, v. 41, n. 3, p. 507-532, 2004.

MOREIRA, I. M.; CORSO, G. Salvador: transformações na ordem urbana [recurso eletrônico]: metrópoles: território, coesão social e governança democrática. / organização Inaiá Maria Moreira de Carvalho, Gilberto Corso Pereira ; coordenação Luiz Cesar de Queiroz Ribeiro. 1. ed. Rio de Janeiro: Letra Capital: Observatório das Metrópoles, 2014.

NAVARRO, C.J.; CLARK, T. N.; SILVER, D.; DÍAZ, P.; GUERRERO, G.; MUÑOZ, L.; RODRÍGUEZ, M.; HUETE, H; MARINERO, R.; MATEOS, C. Las dimensiones culturales de la ciudad, La Catarata, Madrid, 2012.

OLIVEIRA, M. R. N. A Centralidade do Iguatemi. In: Nascimento, Jaime; Gama, Hugo;. (Org.). A Urbanização em Salvador em Três Tempos: Colônia, Império e República. 1ed.Salvador - Bahia: Instituto Geográfico e Histórico da Bahia - IGHB, v. 2, p. 329-373, 2011.

PONZINI, D. y ROSSI, U. Becoming a creative city: The entrepreneurial mayor, network politics and the promise of an urban renaissance. Urban Studies, v. 47, n. 5, p. 1037-1057, 2010 .

DRd - Desenvolvimento Regional em debate (ISSNe 2237-9029) 
PRATT, A. C. y HUTTON, T. Reconceptualising the relationship between the creative economy and the recession: learning from the financial crisis'. Cities, n. 33, p. 86-95, 2013.

PRADA, J. El debate de la creatividad y la economía en las ciudades actuales y el papel de los diferentes actores: algunas evidencias a partir del caso de estudio de Madrid. Revista Investigaciones Geográficas, Boletín del Instituto de Geografía, UNAM, ISSN 0188-4611, n. 87, p. $62-75,2013$.

RALLET, A. Ressources spécifiques et ressorces g'enériques: une problématique pour le développment loca. In: ABDELMAKI, L.; COURLET, C. (Eds). Les nouvelles logiques du développement. París, L’Harmattan, 1995. p. 39- 57

REID, B; ALBERT, A; HOPKINS, L. A Creative Block? The Future of the UK Creative Industries, The Work Foundation. 2010. www.theworkfoundation.com

RHODES, R. Understanding governance: policy networks, governance and reflexivity, Open University Press, Londres, 1997.

SANCHEZ, M. V.; RIUS, J., ZARLENGA, M. Ciudad creativa y ciudad sostenible?: Un análisis crítico del "modelo Barcelona" de políticas culturales. Revista Crítica de Ciências Sociais, n.. 99, p. 31-50, 2012 (Ejemplar dedicado a: Em torno da cidade criativa).

SANTOS, B. DA SOUSA. Desigualdad, Exclusión y Globalización: Hacia la Construcción Multicultural de la Igualdad y la Diferencia. La Caída del Angelus Novus: Ensayos para una Nueva Teoría Social y una Nueva Práctica Política, Bogotá, ILSA, Universdad Nacional de Colombia, 2003. p. 125-165

SANTOS, D. V. C; ANDRADE F.; FREITAS, I. M. D. P. Evolução das centralidades e os impactos sobre a mobilidade na cidade do Salvador. XVI Congresso Pan-Americano de Engenharia de Tráfego e Transportes e Logística, Lisboa, Portugal. 2010.

SANTOS, J. A reestruturação da cidade de Salvador: Conflitos e interesses na lógica da centralidade urbana. GeoTextos, v. 6, n. 1, p. 13-33, jul. 2010.

SCOTT, A. (2006), Creative cities: Conceptual issues and policy questions, Journal of Urban Affairs, Wiley, USA, v. 28, n. 1, p. 1-17, 2006.

SERPA, A. Periferização e metropolização no Brasil e na Bahia: O exemplo de Salvador. GeoTextos, v. 3, n. 1 y 2, p. 31-46 2007.

SIERRA, F. Política cultural y economía creativa en Brasil. Una perspectiva crítica de la cultura para el desarrollo local. Telos: Cuadernos de comunicación e innovación, p. 35-44, 2014 (Ejemplar dedicado a: La publicidad ante el reto digital).

SOLÀ, A. “La traducción de governance" [en línea]. Punto y coma. v. 65, 2000.

Disponible:ettp://ec.europa.eu/translation/bulletins/puntoycoma/65/pyc652.htmpuntoycoma/6 5/pyc652.htm (consulta: junio 2015). 
SUBIRATS, J. Si la respuesta es gobernanza, ¿cuál es la pregunta? Factores de cambio en la política y en las políticas. Ekonomiaz: Revista vasca de economía, n.. 74, p. 16-35, 2010 (Ejemplar dedicado a: Gobernanza y competitividad territorial).

UNCTAD. Creative Economy Report. 2013. Nueva York, Naciones Unidas.

UNCTAD. Creative Economy Report. 2010. Nueva York, Naciones Unidas.

UNCTAD. Creative Economy Report. 2008. Nueva York, Naciones Unidas.

VICARIO, L.; RODRÍGUEZ, A. Innovación, Competitividad y Regeneración Urbana: los espacios retóricos de la "ciudad creativa" en el nuevo Bilbao. Ekonomiaz: Revista vasca de economía, ISSN 0213-3865, n. 58, p. 262-295, 2005 (Ejemplar dedicado a: Ciudades región globales. Espacios creativos y nueva gobernanza).

Artigo recebido em: 07/10/2015

Artigo aprovado em: 09/10/2015 